\title{
Preparation of PAAc Grafted ePTFE Films Immobilized with Enzymes Using for DDS
}

\author{
Kiyomi Matsuda, Yasuhiro Takhaashi, Ayumi Kashiwada, Kazunori Yamada, \\ and Mitsuo Hirata \\ Department of Applied Molecular Chemistry, College of Industrial Technology, \\ Nihon University, \\ 1-2-1 Izumi-cho, Narashino-shi, Chiba 275-8575, Japan
}

\begin{abstract}
The ePTFE-g-PAAc films that were able to supply the insulin of the diabetic medicine near the human blood $\mathrm{pH}$ value were prepared by the combined use of oxygen plasma treatment and AAc photografting at lowered photo-polymerization temperatures compared with the previous report [1-3].

Two enzymes, GOD and Catalase, were immobilized in the ePTFE-g-PAAc films with the same condensation method as before [1-3]. Although the ePTFE-g-PAAc-i-GOD\&Catalase films prepared from AAc-grafting at $60^{\circ} \mathrm{C}$ were permeable to insulin molecules in a buffer solution of $\mathrm{pH} 7.40$, the ePTFE-gPAAc-i-GOD\&Catalase films prepared at $40{ }^{\circ} \mathrm{C}$ considerably obstructed the passage of the insulin molecules. Addition of glucose to the solution leading to the lowering $\mathrm{pH}$ level facilitated the permeation of the insulin molecules through the ePTFE-g-PAAc-iGOD\&Catalase films. The immobilization of the enzymes was also confirmed by IR-ATR and SEM.
\end{abstract}

Keywords: ePTFE-g-PAAc film, immobilization of enzymes, insulin permeation, DDS for diabete

\section{Introduction}

The development of the artificial internal organs that are gentle and safe to the human organisms is urgently needed. Various stimulus responsive materials have been devised for the time regulation and self-control type devices [4, 5], which have been occupied by medical scientists and engineers.

An expanded poly (tetrafluoroethylene) (ePTFE) films we selected as a common polymer substrate, which is known to be medical materials, has thermo-stability, chemical resistance, etc., but can be relatively easily activated by the oxygen plasma preprocessing because of the surface porosity, and so acrylic acid (AAc) monomers can further be adapted to the porous surfaces by photografting polymerization.

The enzymes, GOD and Catalase, were immobilized on poly (acrylic acid) (PAAc) chains grafted on ePTFE film surfaces through the covalent binding method. We have used this film as the stimulus responsive one that can detect glucose: gluconic acid formation from glucose by catalytic action of GOD, reducing the $\mathrm{pH}$ level of the solution around the film surface. PAAc grafted chains on the surface of pores start to contract in response to the $\mathrm{pH}$ lowering, and this results in film pores being opened and, therefore, the permeability of insulin molecules increasing.

The glucose reacts with GOD to form hydrogen peroxide $\left(\mathrm{H}_{2} \mathrm{O}_{2}\right)$ molecules, toxic to living organisms. Then Catalase is expected to work effectively: first, it can consume the $\mathrm{H}_{2} \mathrm{O}_{2}$ generated together with the gluconic acid, and secondly, it can promote the oxidation of glucose through the cogeneration of oxygen.

In this research, the immobilization of GOD and Catalase onto the surface of the ePTFE-g-PAAc film was carried out simultaneously.

\section{Experimental}

2.1 Preparation of ePTFE-g-PAAc films 
The polymer substrate used here, ePTFE film (average pore size $=0.5 \mu \mathrm{m}$, degree of vacancy $=$ $78 \%$, and thickness $=65 \mu \mathrm{m}$, ADVANTEC Co. Ltd.), was cut into circular pieces of a suitable size (47 $\mathrm{mm}$ in diameter) for permeation experiments, followed by washing in methanol and acetone for $10 \mathrm{~min}$ in an ultrasonic washing machine, and then drying under reduced pressure. AAc (Wako Pure Chemical Ind., Ltd.) as a hydrophilic monomer and a benzophenone (Wako Pure Chemical Ind. Ltd.) as a sensitizer were used as received without further purification. The oxygen plasma treatment was performed to one side of each ePTFE film for 2 min by the use of a Shimadzu LCDV-20 type plasma polymerization apparatus, and the plasma-treated ePTFE films were dipped for 1 min in a similar fashion to the previous procedure in our previous articles [1-3]. After drying, the sensitizer-coated ePTFE films were put into the Pyrex glass tube containing an aqueous monomer solution of $50 \mathrm{~cm}^{3}$ of $2.0 \mathrm{~mol} / \mathrm{dm}^{3}$ and then photo-polymerized at 40 or $60 \square \mathrm{C}$ for 2 to 7 hours by the use of a Riko rotary photochemical reactor RH400-10W (Riko Kagaku Ind.) equipped with a 400W high-pressure mercury lamp [1-3].

\subsection{Immobilization of enzymes onto the ePTFE-g-PAAc film}

The co-immobilization of GOD and Catalase was carried out by the condensation reaction with 1-aminohexyl-3-(2-morpholinoethyl) carbodiimido methoxy- $p$-toluene sulfonate (CMC) [1-3, 6, 7].

\subsection{Glucose responsiviness of immobilized enzymes}

The enzymatic activity of immobilized GOD onto the ePTFE-g-PAAc films was followed up by detection of the remaining glucose with the F-kit glucose (Beringer Mannheim Co. Ltd.). The enzymatic activity of immobilized Catalase was evaluated from the absorbance of the color developed by 4-aminoantipyrine, phenol, and peroxidase at $505 \mathrm{~nm}$.

2.4 Infrared spectrum analysis of the ePTFE-g-PAAc-i-GOD\&Catalase film

The ATR spectra of ePTFE film, the ePTFE-g-PAAc film, and the ePTFE-g-PAAc-iGOD\&Catalase film were recorded on the Fourier transform infrared spectrophotometer (FT/IR-4200A; JASCO Co. Ltd.), using the non-polarized light infrared rays and multiplication frequency of 100 times. The scanning speed and the spectral resolution of the DTGS (Duteriated Triglycine Sulfate) detector

were set at $2 \mathrm{~mm} / \mathrm{s}$ and to $4 \mathrm{~cm}^{-1}$, respectively.

2.5 Observation of surface of the ePTFE-gPAAc-i-GOD\&Catalase film by the scanning electron microscope

The surface morphology of the ePTFE-gPAAc-i-GOD\&Catalase film was analyzed by the scanning electron microscope (SEM) (VE-9800; Keyence Co. Ltd.) on the conditions of an acceleration voltage of $5 \mathrm{kV}$, a magnification of 4000 times and a resolution of $8 \mathrm{~nm}$.

\subsection{Permeation Control of Insulin}

The ePTFE-g-PAAc-i-GOD\&Catalase films were clamped between the two halves of the cell as shown in Fig. 1[3,5] in order to measure the amount of permeated insulin. The permeation side cell contained only a phosphate buffer solution adjusted to $\mathrm{pH} 7.40$ and the feed side cell the phosphate buffer solution of the same $\mathrm{pH}$ as the permeation side, including insulin $\left(1.0 \times 10^{-5}\right.$ $\mathrm{mol} / \mathrm{dm}^{3}$ ). The absorbance of the solution in the permeation side was measured at $275 \mathrm{~nm}$ every 30 minutes, and so the amount of permeated insulin was spectrophotometrically calculated. The permeation control of insulin was preliminarily checked as follows: First, a phosphate buffer solution of $\mathrm{pH} 6.50$ was added to the permeation side cell to confirm the permeation of the insulin in the contraction state of the PAAc grafted chains of the ePTFE-g- PAAc-i-GOD\& Catalase film. Secondly, to confirm the glucose-responsiviness, $0.18 \mathrm{~g}$ of glucose was added to the permeation side cell 90 minutes after the beginning of the experiment on the permeation of the insulin

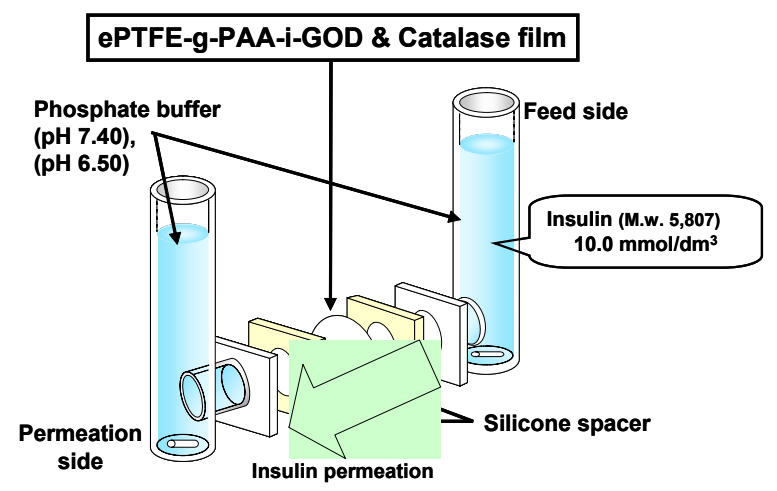

Fig. 1 Schematic diagram of permeation experiments 


\section{Results and Discussion}

\section{1 ePTFE-g-PAAc films}

The relation between the grafting ratio and reaction time of preparing ePTFE-g-PAAc-films is shown in Fig. 2. The grafting ratio was calculated from the following equation.

The grafting ratio $=\mathrm{Wg} / \mathrm{Wo}$

$\mathrm{Wg}$ : the weight of the ePTFE-g-PAAc film

Wo: the weight of the untreated ePTFE film

The grafting ratios from photografting polymerization at $40{ }^{\circ} \mathrm{C}$ and $60{ }^{\circ} \mathrm{C}$ were constant over the grafting time in this study. However, in case ePTFE-g-PAAc films were again AAcpolymerized at the same conditions as before, the grafting ratio increased up to 3 times as much as the ePTFE-g-PAAc films [3].

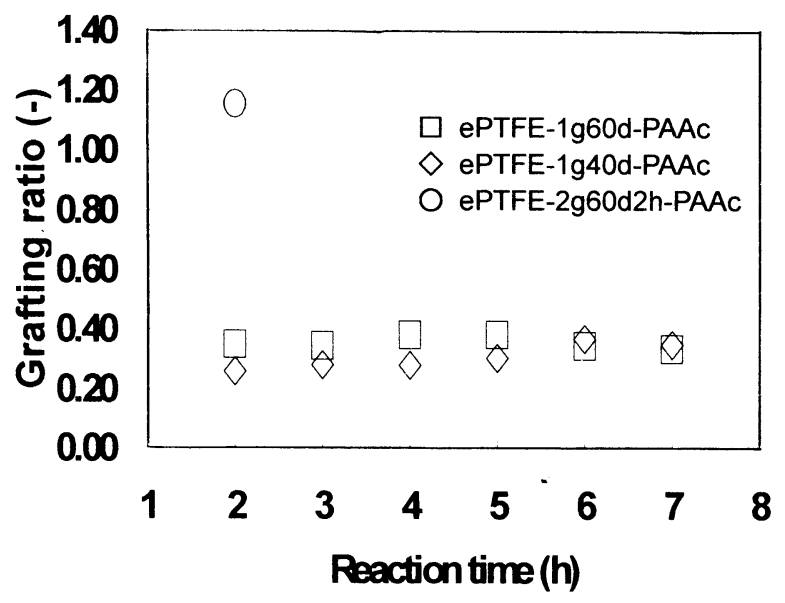

Fig. 2 Relation between grafting ratio and reaction conditions

3.2 Enzymatic activities of co-immobilized GOD and Catalase

To investigate the activities of the immobilized GOD, ePTFE-g-PAAc-i-GOD\&Catalase films were immersed in a glucose solution and the change in the concentration of the residual glucose with time were measured with that of native GOD. It was confirmed from Fig. 3 that the enzymatic activities of GOD immobilized on the ePTFE-g-PAAc-iGOD\&Catalase films considerably persisted, with the decreased activities compared to native GOD.

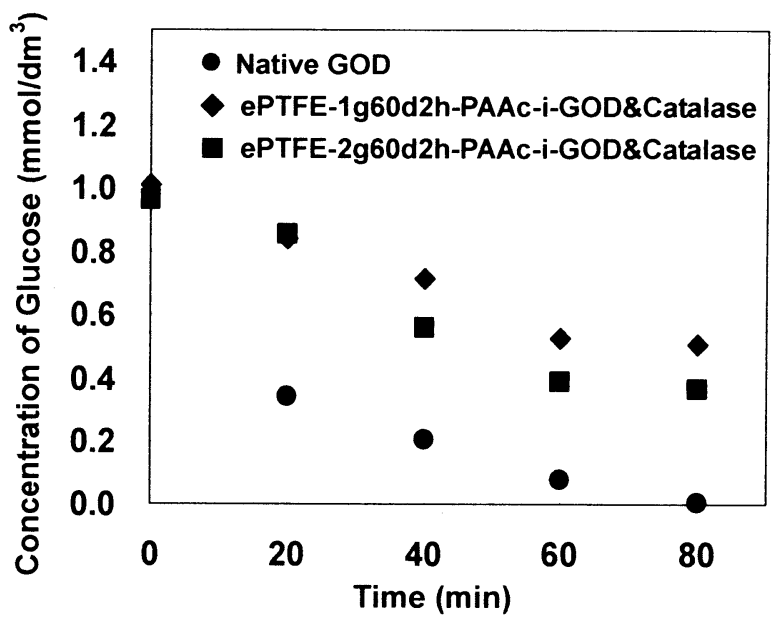

Fig. 3 Determination of enzyme activity of GOD

3.3 Infrared spectrum analysis on the surface of ePTFE-g-PAAc-i-GOD\&Catalase films

The infrared-ATR spectra on the surface of the ePTFE (untreated ePTFE), the ePTFE-g-PAAc, and the ePTFE-g-PAAc-i-GOD\&Catalase films are shown in Fig. 4. In the untreated ePTFE film, only two sharp peaks of C-F stretching were observed around $1200 \mathrm{~cm}^{-1}$.

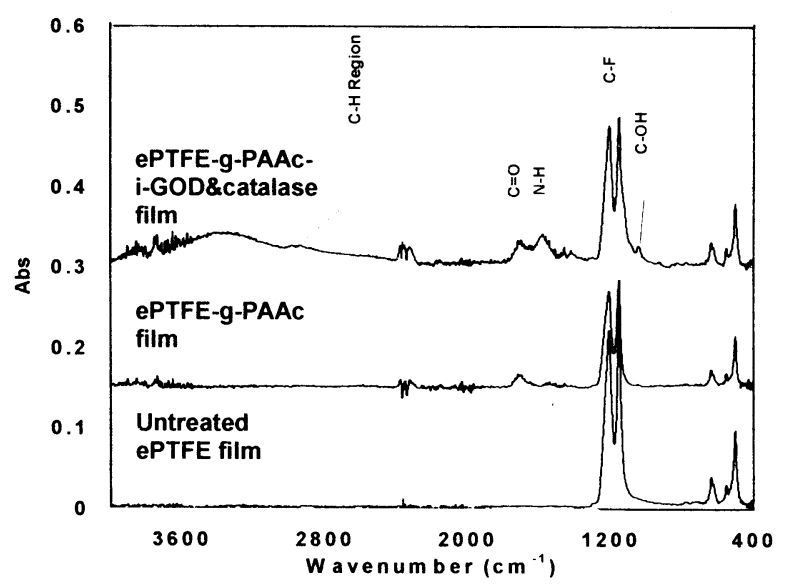

Fig. 4 IR-ATR spectra of untreated ePTFE, ePTFE-g-PAAc and ePTFE-gPAAc-i-GOD\&Catalase films 
For the ePTFE-g-PAAc film, a small peak in the $\mathrm{C}-\mathrm{H}$ area around $2900 \mathrm{~cm}^{-1}$ and a peak of $\mathrm{C}=\mathrm{O}$ at $1700 \mathrm{~cm}^{-1}$ were confirmed. These peaks are assigned to the PAAc origin. Finally, for the ePTFE-g-PAAc-i-GOD\&Catalase film, the O-H stretching band around $3400 \mathrm{~cm}^{-1}$, the $\mathrm{C}-\mathrm{H}$ region around $2900 \mathrm{~cm}^{-1}$, N-H stretching band around $1600 \mathrm{~cm}^{-1}$, and the peak of $\mathrm{C}-\mathrm{OH}$ were detected. These peaks are assigned to the amino acid origin that support the presence of the protein, that is, the enzyme.

The peaks around $1700 \mathrm{~cm}^{-1}$ could be attributed to the $\mathrm{C}=\mathrm{O}$ bond, and the peak around $1600 \mathrm{~cm}^{-1}$ to the $\mathrm{N}-\mathrm{H}$ bond. They are originated from the amide bond. From the spectral results described above, it is clear that the enzymes were immobilized on the ePTFE-g-PAAc-i-GOD\&Catalase film.

\subsection{Observation of ePTFE-g-PAAc-i-GOD\&} Catalase film by SEM

The SEM photograph of the ePTFE-g-PAAc-iGOD\&Catalase film is shown in Fig. 5.

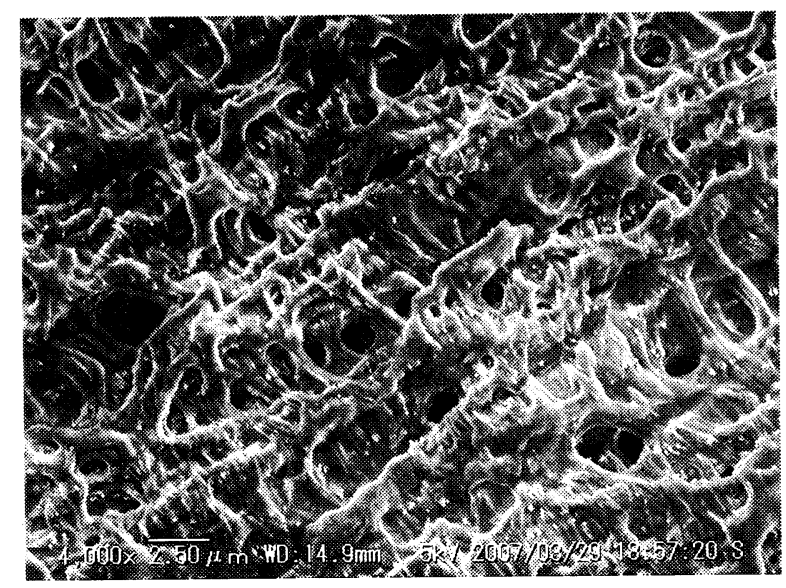

Fig. 5 SEM picture of ePTFE-gPAAc-i-GOD\&Catalase film surface

The white wide fibers with highly crystalline parts of ePTFE were covered with grafted PAAc chains, but a lot of pores appear yet to be packed with PAAc chains. It is thought that the grafted PAAc chains were forced to severely shrink because the SEM photograph was taken in vacuum, leading to removal of water molecules around grafted PAAc chains.
3.5 Permeation control of insulin by ePTFE-g-PAAc-i-GOD\&Catalase film

Fig. 6 shows the change in the amount of permeated insulin in a phosphate buffer solution of $\mathrm{pH} 7.40$, near to the $\mathrm{pH}$ of human blood, through the ePTFE-g-PAAc-i-GOD\&Catalase film with time. The ePTFE-g-PAAc film utilized here had a high grafted ratio prepared by grafting twice. The permeation experiments using this film revealed that a considerable amount of insulin passed through the film. However, this film suppressed the insulin permeation in a buffer solution of $\mathrm{pH} 7.80$, and, therefore, this film was not in the sufficient swelling state to obstruct the passage of insulin molecules in the buffer solution of $\mathrm{pH} 7.40$.

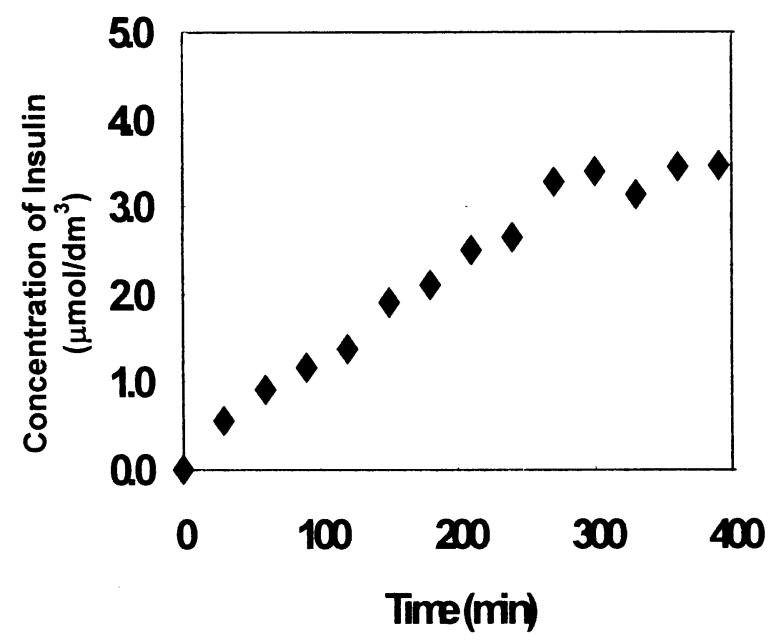

Fig. 6 Changes in the Insulin perme- abilities through ePTFE-2g60d2hPAAc-i-GOD\&Catalase film in $\mathrm{pH} 7.40$ phosphate buffer solution

Therefore, since polymer chain length increases with the decrease in the polymerization temperature according to the polymerization kinetics, the temperature of the photografting was lowered to $40^{\circ} \mathrm{C}$. Fig. 7 shows the insulin permeation behavior through the ePTFE-g-PAAc-i-GOD\& Catalase films prepared for 6 hours at $40{ }^{\circ} \mathrm{C}$ and $60{ }^{\circ} \mathrm{C}$. The film prepared for 6 hours at $40{ }^{\circ} \mathrm{C}$ suppressed the permeation of insulin compared with the films prepared for 6 hours at $60^{\circ} \mathrm{C}$. It is considered that PAAc chains grafted at $40{ }^{\circ} \mathrm{C}$ became longer than those at $60{ }^{\circ} \mathrm{C}$ as expected and filled up pores effectively. 
Though the amount of PAAc grafted ratio decreased when polymerization temperature was lowered from $60^{\circ} \mathrm{C}$ to $40{ }^{\circ} \mathrm{C}$, we found from the results shown in Fig. 7 that the longer grafted chains were able to close pores more effectively even if the grafted ratio was low.

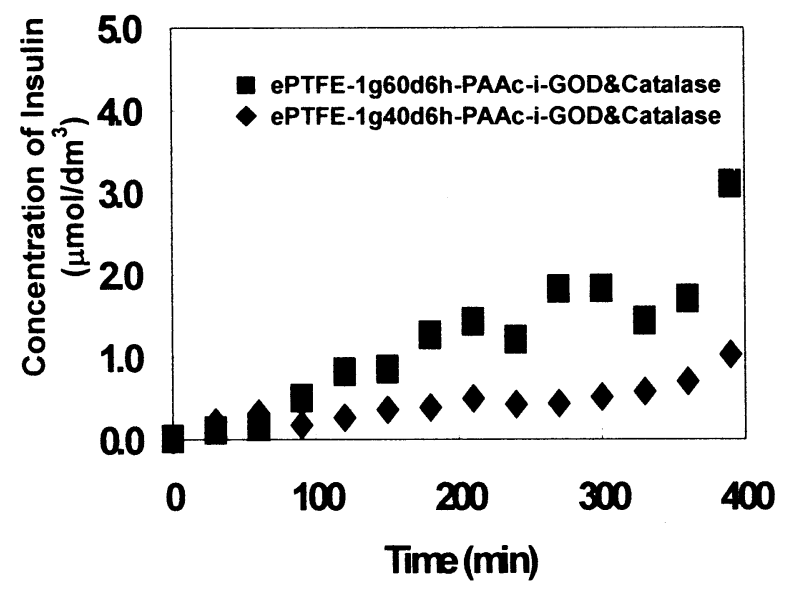

Fig. 7 Changes in the Insulin perme- abilities through ePTFE-(1g40d6h, 1 g60d6h)-PAAc-iGOD\&Catalase films in $\mathrm{pH} 7.40$ phosphate buffer solution

To confirm the enhanced permeation of insulin due to the $\mathrm{pH}$ lowering by using the film prepared at $40{ }^{\circ} \mathrm{C}$, the permeation experiment of insulin was carried out in a buffer solution of $\mathrm{pH} 6.50$.

The insulin permeation at $\mathrm{pH}$ values of 6.50 and 7.40 through ePTFE-1g40d6h-PAAc-i-GOD\& Catalase films is shown in Fig. 8.

Because insulin permeated through the film in pH 6.50 buffer solution, it was understood that the ePTFE-g-PAAc-i-GOD\&Catalase film had gained the $\mathrm{pH}$ lowering response. The grafted ratio of PAAc decreased because of polymerization temperature lowering from $60{ }^{\circ} \mathrm{C}$ to $40{ }^{\circ} \mathrm{C}$.

Finally, we carried out the permeation control of insulin due to the $\mathrm{pH}$ change induced by enzymatic reactions of GOD and Catalase. The result is shown in Fig. 9. The ePTFE-gPAAc-i-GOD\&Catalase film in a buffer solution of pH 7.40 suppressed the insulin permeation for 90 min. After the addition of glucose into the permeation side cell at $90 \mathrm{~min}$, the amount of permeated insulin increased gradually.

Since the PAAc chains of the ePTFE-g-PAAc-

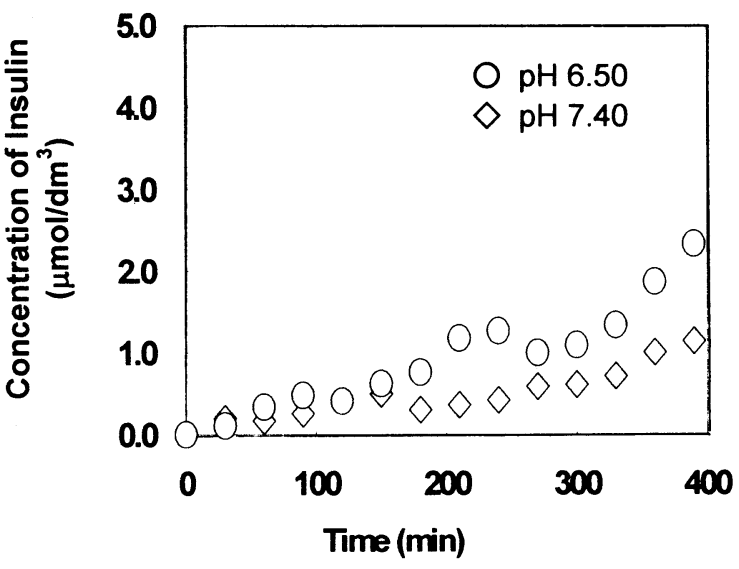

Fig. 8 Changes in the Insulin permeabilities through ePTFE-1g40d6h- PAAc-i-GOD\&Catalase film in $\mathrm{pH} 6.50$ and $\mathrm{pH} 7.40$ phosphate buffer solutions

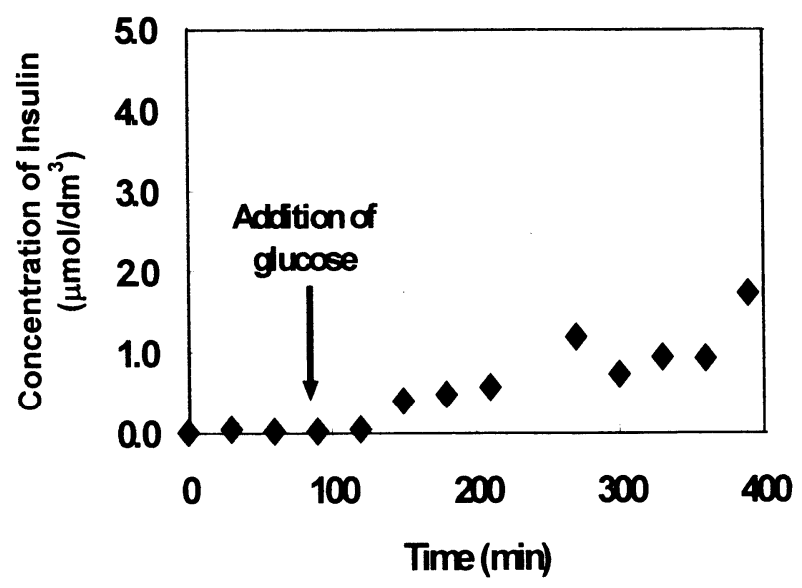

Fig. 9 Changes in the Insulin perme- abilities through ePTFE-1g40d6h-PAAc-i- GOD\&Catalase film in $\mathrm{pH} 7.40$ phosphate buffer solution

i-GOD\&Catalase film used here can expand in the phosphate buffer solution of $\mathrm{pH} 7.40$ by the electrostatic repulsion between carboxyl anions, and so make the channels in the films narrow, the insulin permeation is considered to be depressed.

The permeation of insulin gradually happened 30 minutes after addition of glucose. It is considered that gluconic acid generated by the enzymatic reaction between GOD and glucose declines $\mathrm{pH}$ around the surface of the film and prevents carboxyl groups from dissociating. As a 
result, suppression of electrostatic repulsion among the grafted PAAc chains makes the channels open so that the insulin moves to the permeation side cell gradually. Further, the amount of permeated insulin increased a little by the ePTFE-gPAAc-i-GOD\&Catalase film that made enzyme GOD and Catalase immobilized at the same time.

\section{Conclusions}

For the construction of the DDS system for diabetes, we prepared ePTFE films with grafted PAAc and immobilized GOD and Catalase onto the PAAc chains. In an attempt to construct the DDS system, the permeation experiments were carried out under the condition set to $\mathrm{pH} 7.40$ near human blood $\mathrm{pH}$. From the results, we can draw the following conclusions.

1) The addition of glucose to the system suggested here facilitated the permeation of insulin through the ePTFE-g-PAAc-i-

2) GOD\& Catalase film.

3) A DDS system was constructed with high potentiality to be used gently and safely.

\section{Acknowledgments}

This work was partly supported by High Tech. Research Center of College of Industrial Technology, Nihon University.

\section{References}

1) K. Matsuda, H. Matsuzaka, K. Yamada, and M. Hirata, J. Photopolym. Sci., Tech., 14. (2001) 81

2) K. Matsuda, T. Makino, A. Kashiwada, K. Yamada, and M. Hirata, J. Photopolym. Sci., Tech., 17. (2004) 165

3) K. Matsuda, Y. Kawahara, S. Shimada, A. Kashiwada, K. Yamada, and M. Hirata, $J$. Photopolym. Sci., Tech., 19. (2006) 225

4) K. Ishihara, K. Matsui, J. Polym. Sci., 24(1986) 413

5) Kai Zhang, Xiao Yu Wu, J. Control. Release, 80 (2002) 169

6) Lei Ying, E. T. Kang, K. G. Neoh, J. Membrane Sci., 208 (2002) 361

7) K. Podual, F. J. Doyle III, N. A. Peppas, J. Controlled Release, 67 (2000) 9 